Research Article

\title{
Karyotypic evolution of ribosomal sites in buffalo subspecies and their crossbreed
}

Tiago Marafiga Degrandi ${ }^{1}$, Sebastian Pita $^{2}$, Yanina Panzera ${ }^{2}$, Edivaldo Herculano C. de Oliveira ${ }^{3,5}$, José Ribamar Felipe Marques ${ }^{4}$, Marivaldo Rodrigues Figueiró ${ }^{4}$, Larissa Coêlho Marques ${ }^{5}$, Lucia Vinadé ${ }^{1}$ Ricardo José Gunski ${ }^{1}$ and Analía Del Valle Garnero ${ }^{1}$

${ }^{1}$ Universidade Federal do Pampa, Campus São Gabriel, São Gabriel, RS, Brazil.

${ }^{2}$ Faculty of Science, University of the Republic, Montevideo, Uruguay.

${ }^{3}$ Laboratório de Cultura de Tecidos e Citogenética, Institute Evandro Chagas, Ananindeua, PA, Brazil.

${ }^{4}$ Empresa Brasileira de Pesquisa Agropecuária, Amazônia Oriental, Belém, PA, Brazil.

${ }^{5}$ Instituto de Ciências Exatas e Naturais, Faculdade de Ciências Naturais, Universidade Federal do Pará, Belém, PA, Brazil.

\begin{abstract}
Domestic buffaloes are divided into two group based on cytogenetic characteristics and habitats: the "river buffaloes" with $2 n=50$ and the "swamp buffaloes", $2 n=48$. Nevertheless, their hybrids are viable, fertile and identified by a $2 n=$ 49. In order to have a better characterization of these different cytotypes of buffaloes, and considering that NOR-bearing chromosomes are involved in the rearrangements responsible for the karyotypic differences, we applied silver staining (Ag-NOR) and performed fluorescent in situ hybridization (FISH) experiments using 18S rDNA as probe. Metaphases were obtained through blood lymphocyte culture of 21 individuals, including river, swamp and hybrid cytotypes. Ag-NOR staining revealed active NORs on six chromosome pairs $(3 p, 4 p, 6,21,23,24)$ in the river buffaloes, whereas the swamp buffaloes presented only five NOR-bearing pairs $(4 p, 6,20,22,23)$. The F1 crossbreed had 11 chromosomes with active NORs, indicating expression of both parental chromosomes. FISH analysis confirmed the numerical divergence identified with Ag-NOR. This result is explained by the loss of the NOR located on chromosome $4 \mathrm{p}$ in the river buffalo, which is involved in the tandem fusion with chromosome 9 in this subspecies. A comparison with the ancestral cattle karyotype suggests that the NOR found on the $3 p$ of the river buffalo may have originated from a duplication of ribosomal genes, resulting in the formation of new NOR sites in this subspecies.
\end{abstract}

Keywords: 18S rDNA, Bubalus bubalis, FISH.

Received: October 29, 2013; Accepted: March 26, 2014.

\section{Introduction}

The Bovidae family (Mammalia, Artiodactyla) comprises almost 140 species, usually divided phylogenetically into ten subfamilies, from which Bovinae (tribes Bovini, Boselaphini and Tragelaphini) includes the most economically important species on the planet. The domestic buffaloes and cattle are included in the Bovini tribe, in the subtribe Bubalina and Bovina, respectively. Molecular studies have inferred that these two subtribes had a monophyletic origin with cattle having a more recent origin. Nevertheless, many Bovini species have uncertain interespecific phylogenetic relationships due to their short period of evolutionary divergence (MacEachern et al., 2009).

Send correspondence to Tiago Marafiga Degrandi. Universidade Federal do Pampa, Campus São Gabriel, Avenida Antônio Trilha 1847, 97300-000 São Gabriel, RS, Brazil. E-mail: t.degrandi @yahoo.com.br.
According to cytogenetic studies, the karyotype of the cattle, $2 \mathrm{n}=60(\mathrm{FN}=58)$, is considered as the ancestral model for evolutionary comparisons with other Bovidae species due to its predominance of telocentric chromosomes, a karyotypic formula observed in various species (Wurster and Benirschke, 1968). The evolutionary history of this family is characterized by few rearrangements, comprising mainly centric fusions between uniarmed chromosomes resulting in derived biarmed chromosomes, such as observed in the domestic buffaloes (Di Berardino et al., 1985; Di Meo et al., 1991, 1993).

Two subspecies of domestic buffaloes (Bubalus bubalis) are recognized based on cytogenetic differences and adaptation to different habitats: The first group is denominated "river buffaloes" (B. bubalis bubalis) and presents $2 \mathrm{n}=50(\mathrm{FN}=58)$, and the second group, the "swamp buffaloes" (B. bubalis Kerebau), have $2 \mathrm{n}=48(\mathrm{FN}=56)$ and is represented by the Carabao breed (Bongso and Hilmi 
1982; Iannuzzi et al., 1996). There is also the wild Tamaraw buffalo ( $B$. mindorensis) that has $2 \mathrm{n}=46(\mathrm{FN}=56)$ (Tanaka et al., 2000).

Comparisons between the GTG and RBG-banded karyotypes of river buffaloes and catlle indicated that the five larger biarmed chromosome pairs of the river buffalo were homologous to cattle chromosomes 1/25, 2/23, 8/19, $5 / 28$ and $16 / 29$, from which they originated by centric fusions (Iannuzzi et al., 1990). On the other hand, swamp buffaloes diverged from river buffaloes by a tandem fusion between chromosomes 4 and 9, which gave rise to the largest chromosome pair in the first subspecies (Di Berardino et al., 1981; Bongso and Hilmi, 1982; Iannuzzi and Di Berardino, 1985).

Analyses of the nucleolus organizer regions (NORs) indicated that species of the Bovidae family have ten highly conserved NOR chromosomal sites (Di Berardino et al., 1985; Di Meo et al., 1991, 1993). However, differences in the number of NOR-bearing chromosomes were observed in domestic buffaloes. The river buffalo (B. bubalis bubalis) showed NORs in six chromosome pairs (3p, 4p, 6 , 21, 23 and 24), whereas the swamp buffalo (B. bubalis Kerebau) had five NOR-bearing pairs (4p, 6, 20, 22 and 24) (Di Berardino et al., 1981; Hilmi and Bongso, 1983; Iannuzzi et al., 1990, 1996; Tanaka et al., 2000). Despite differences between karyotypes of the river and swamp buffaloes, these animals are not reproductively isolated and the F1 crossbreed, with $2 \mathrm{n}=49$, is viable and fertile.

The domestic buffaloes are economically important for the development of various countries. In Brazil, they were introduced around 1889, initially in the Marajó Island, where the buffaloes of the Baio type and the Carabao breed represent the river and swamp group, respectively. The populations of these animals are small and their hybridization threatens their genetic inheritance with racial miscegenation (Marques et al., 2003).

Because NOR-bearing chromosomes are involved in the rearrangements responsible for the karyotypic differences in the buffaloes races, we aimed to compare the karyotypes of these breeds and of the hybrids using silver staining of the NORs (Ag-NORs) and FISH with $18 \mathrm{~S}$ rDNA probes in order to understand the dynamic of activation and relocation of these sites in the karyotypes of buffaloes during their divergence and hybridization.

\section{Materials and Methods}

\section{Samples and cell culture}

Blood samples from ten Baio type (river buffalo) specimens, ten Carabao breed individuals (swamp buffalo) and one female crossbreed F1 were collected in heparinized syringes. Samplings were conducted in accordance to the rules of the institutional committee for the project "in situ" Conservation of Animal Genetic Resources from Brazil; Large Scale Species" Security Code 01.06.01.06.00.04/EM-
BRAPA-Eastern Amazon. Lymphocyte cultures were performed according to Moorhead et al. (1960). Briefly, $1 \mathrm{~mL}$ of peripheral blood was added to $10 \mathrm{~mL}$ of RPMI $1640 \mathrm{cul}-$ ture medium supplemented with $20 \%$ fetal bovine serum, $0.05 \mathrm{~mL}$ penicillin/streptomycin and $0.1 \mathrm{~mL}$ of phytohemaglutinin, and $0.01 \mathrm{~mL}$ of $0.05 \%$ colchicine was added after $71 \mathrm{~h}$ of incubation. After harvesting, hypotonic solution $(0.75 \mathrm{M} \mathrm{KCl})$ was added and the cells were incubated for $20 \mathrm{~min}$ at $37{ }^{\circ} \mathrm{C}$. Finally, the material was fixed and washed with fixative (3:1 methanol and acetic acid solution).

\section{Sequential analysis and Ag-NORs}

The diploid numbers and chromosome morphologies of each individual karyotype were determined after the analysis of approximately 40 metaphases stained with Giemsa. The representative metaphases were registered using a digital camera and the chromosomes were arranged according to the standard karyotype of river buffaloes (Iannuzzi, 1994). After that the slides were used for sequential Ag-NOR staining, following Howell and Black (1980).

\section{Fluorescence in situ hybridization}

\section{Ribosomal sequence $18 S$}

The $18 \mathrm{~S}$ ribosomal probe from the $45 \mathrm{~S}$ sequence of Bubalus bubalis was generated using data available at the National Center for Biotechnology Information (NCBI accession number JN412502.1). The probe was produced by polymerase chain reaction (PCR) with the following primers: forward: TACCTGGTTGATCCTGCCAGTAGCAT ATGC; reverse: GTTCACCTACGGAAACCTTGTTAC GACTT), according to Pang et al. (2012). The probes were directly labeled with dUTP-Cy3. The hybridizations were performed according to de Oliveira et al. (2005) and the chromosomes were counterstained with DAPI. The fluorescent images were observed and photographed under an OLYMPUS BX53 microscope.

\section{Results}

\section{Sequential analysis after Giemsa staining and Ag-NOR}

Buffaloes belonging to the Baio type had $2 n=50$ and NORs were observed on the short arms of two pairs ( $3 p$, $4 \mathrm{p})$, and on the long arms of four chromosome pairs $(6,21$, 23 and 24), always in the terminal region (Figure $1 \mathrm{~A}$ and B). The Carabao breed showed $2 \mathrm{n}=48$ and NORs on five pairs (4p, 6, 20, 22 and 23) (Figure 1C and D). For the F1 crossbreed we found $2 n=49$ with NORs on five pairs ( $3 p$, 6, 20, 22 and 23) and on the chromosome homologous to the Baio type $4 \mathrm{p}$ (Figure $1 \mathrm{E}$ and $\mathrm{F}$ ).

\section{S FISH analysis}

FISH using $18 \mathrm{~S}$ rDNA as probe confirmed the number of NOR-bearing chromosomes observed after silver staining. Thus, the animals of the Baio type had 12 signals 
(on 3p, 4p, 6, 21, 23 and 24) (Figure 2A) and the Carabao breed displayed ten signals (on 4p, 6, 20, 22 and 23) (Figure 2B). Eleven signals were observed in the F1 hybrid (Figure $2 \mathrm{C})$.

\section{Discussion}

According to Iannuzzi et al. (1990), comparative studies of GTG- and RBG-banded karyotypes showed extensively conserved sinteny between the chromosomes of cattle and buffaloes. MacEachern et al. (2009) concluded that the results from mitochondrial sequences analyses also suggested a clear monophyletic origin for cattle and buffaloes, which diverged about 14 million years ago. Nevertheless, a few ambiguities in interspecific relationships, probably due to the short evolutionary time, have been observed in some species of Bovini. The data from chromosomal studies may be used to try to explain the discrepancies of some proposals and to resolve possible ambiguities in the phylogenetic relationships.

The buffalo lineage includes species from the Bubalus and Syncerus genera, whereas the bovine lineage includes the Bos and Bison genera. According to Wurster and Benirschke (1968), the cattle karyotype, $2 \mathrm{n}=60$ and $\mathrm{FN}=58$, can be considered the plesiomorphic ancestral karyotype of the Bovidae family, due to its predominance of telocentric chromosomes, although molecular data suggest that cattle is evolutionarily more recent (MacEachern et al., 2009). For cytogenetic comparisons, we adopted the hypothesis of an ancestral cattle karyotype. Data from the literature indicate that the bubaline lineage had frequent events of centric fusions and Robertsonian translocations, resulting in the formation of biarmed chromosomes.

The river buffaloes have a closer relationship with swamp buffaloes rather than to other members of the same species. The separation between buffaloes may be associated with geographic patterns, as river buffaloes originated in India and swamp buffaloes are generally restricted to East Asia, where hybridization between the two races has been already documented (Bongso and Hilmi, 1982).

Tandem fusions between telocentric chromosomes were the main mechanism of differentiation among the karyotypes of the bubaline linage. For instance, the five pairs of biarmed chromosomes of the river buffalo are homologous to cattle chromosomes $1 ; 25,2 ; 23,8 ; 19,5 ; 28$; 16;29 (Iannuzzi et al., 1990). As a consequence of these fusions the diploid number decreased from $2 n=60$ in cattle to $2 \mathrm{n}=50$ in river buffaloes, but the $\mathrm{FN}=58$ was conserved.

The diploid number difference between the river $(2 \mathrm{n}=50)$ and swamp $(2 \mathrm{n}=48)$ buffaloes was explained by a second event of tandem fusion. This fusion was between the telomere of $4 p$ and the centromere of pair 9 of the river buffalo, resulting in the formation of the largest metacentric (chromosome 1) in the swamp buffalo (Hilmi and Bongso, 1983). Centromere inactivation may have occurred in the process and, although the diploid and fundamental numbers have been reduced ( $2 \mathrm{n}=50$ to 48 and FN from 58 to 56 ), no reproductive isolation was observed between these two buffalo group.

Similarly, the Tamaraw buffalo (Bubalus mindorensis) is distinguishable from the domestic buffalo by the presence of $2 n=46$ and six biarmed chromosome pairs. According to Tanaka et al. (2000), this divergence is due to a tandem fusion between chromosomes $4 \mathrm{p}$ and 12 from the standard karyotype of the river buffalo, and resulted in the largest chromosome pair of the Tamaraw. GTG-banding revealed partial homology of this chromosome to pair 1 of the swamp buffalo, which also shows a fusion between chromosomes $4 p$ and 9 of the river buffalo. The second largest pair of the Tamaraw buffalo originated from the Robertsonian translocation of telocentric pairs 7 and 15 of the river buffalo.

\section{Evolution of the NOR sites in cattle and buffaloes}

NOR sites have been shown to be well conserved in Bovidae species, and are usually found in five chromosome pairs. Divergences were observed in river buffaloes, which showed NORs in six chromosome pairs, whereas swamp and Tamaraw buffaloes presented the usual five pairs, also found in cattle. Considering the ancestral karyotype as that of cattle, it is possible to relate the chromosomal rearrangements that occurred during evolution and the NOR-bearing chromosomes (Figure 3).

The river buffaloes show NORs in six pairs of chromosomes (3p, 4p, 6, 21, 23, 24), from which only the NOR on $3 p$ is not observed in cattle, suggesting that this site was originated from a duplication event of the ribosomal genes and subsequent insertion in the short arm of chromosome 3 . This observation was not advanced by other authors studying NOR sites in these species. The NOR sites on $4 p, 6,21$, 23 and 24 of the river buffaloes are homologous to the cattle NORs on pairs $28,3,22,26$ and 27 , respectively, and were conserved in their telomeric positions.

The NOR-bearing chromosomes are one of the differences between the river and swamp buffaloes karyotypes, with six pairs in the former ( $3 p, 4 p, 6,21,23,24)$, and five pairs in the latter $(4 p, 6,20,22,23)$. This divergence was explained by the tandem fusion $4 \mathrm{p} ; 9$ in river buffaloes, which involved the NOR region (Bongso and Hilmi, 1982). In addition, some pairs were reordered in the karyotype with $2 \mathrm{n}=48$, in which the fused chromosome $4 \mathrm{p} ; 9$, identified as a large metacentric in the swamp buffalo, is the largest chromosome (pair 1) in the karyotype. Accordingly, the NOR-bearing chromosome 3 of the river buffalo corresponds to pair 4 of the swamp buffalo, and chromosomes 21,23 and 24 of the river buffalo are equivalent to pairs 20, 22 and 23, respectively, in the swamp buffalo (Figure 3).

Despite the differences in karyotypes, hybrids between buffaloes are viable and fertile with crossbreed F1 buffaloes with $2 n=49$. The hybrids have 11 NOR-bearing chromosomes, which is the maximum number of NORs ob- 
a)

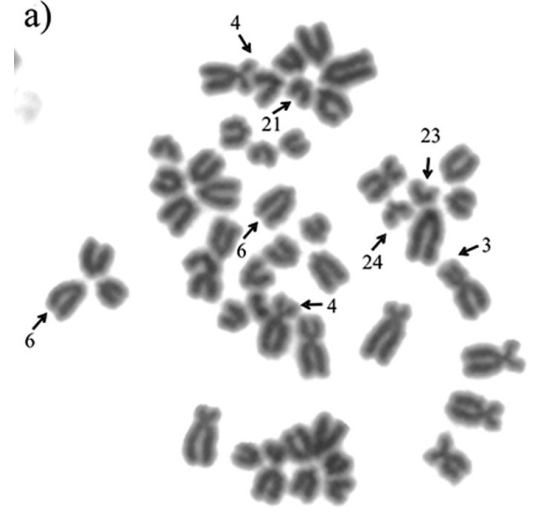

c)

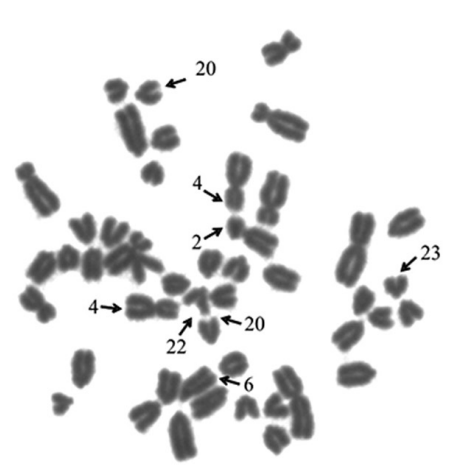

e)

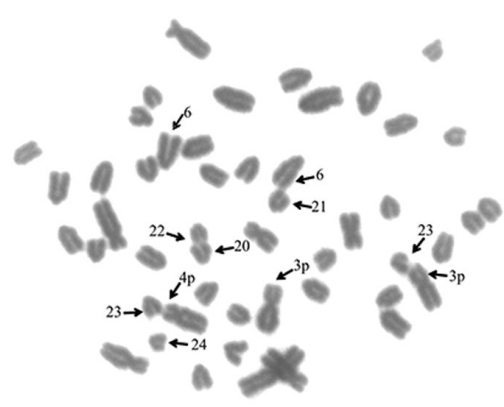

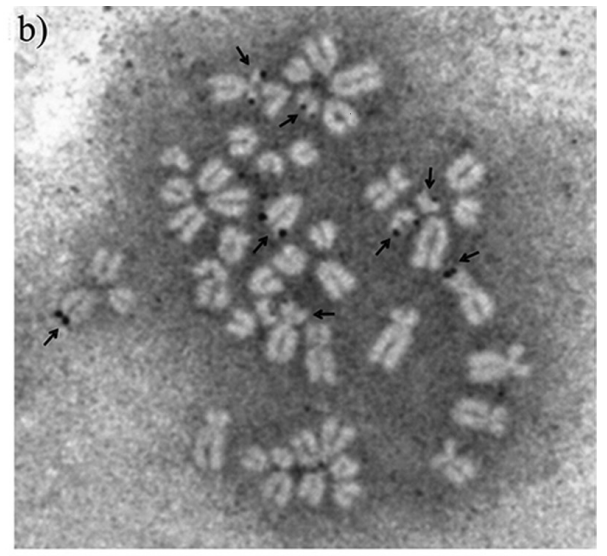
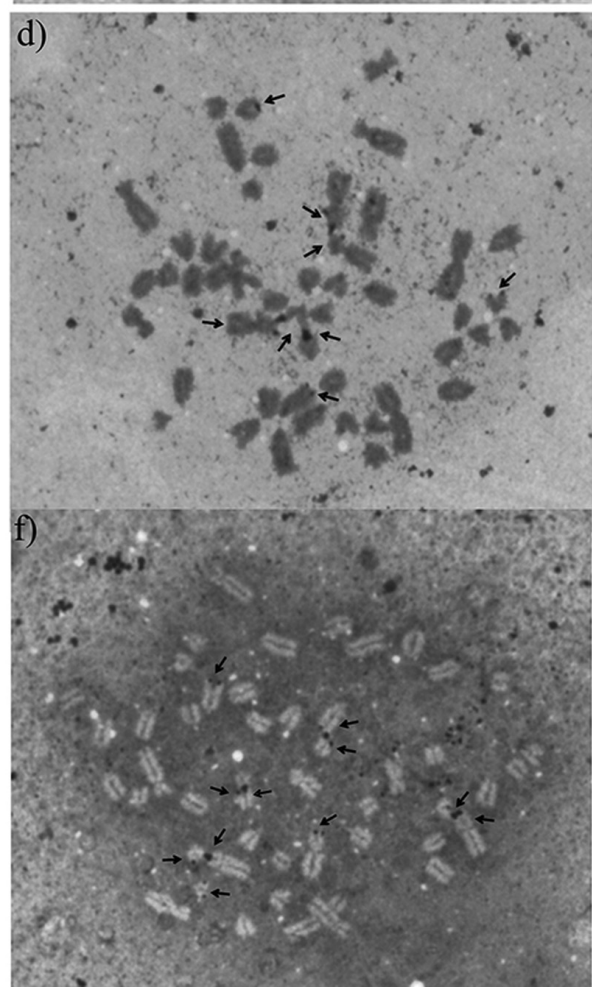

Figure 1 - Sequential Giemsa/Ag-NOR staining in buffalo subspecies and in the F1 crossbreed. In (A) and (B) female of the Baio type ( $2 \mathrm{n}=50)$ with Ag-NORs in chromosomes 3p, 4p, 6, 21, 23 and 24. In (C) and (D) male Carabao breed $(2 n=48)$ with Ag-NORs in 4p, 6, 20, 22 and 23. In (E) and (F) F1 female crossbreed $(2 n=49)$ with 11 Ag-NOR-bearing chromosomes, eight of which show associations. The arrows point the Ag-NORs.

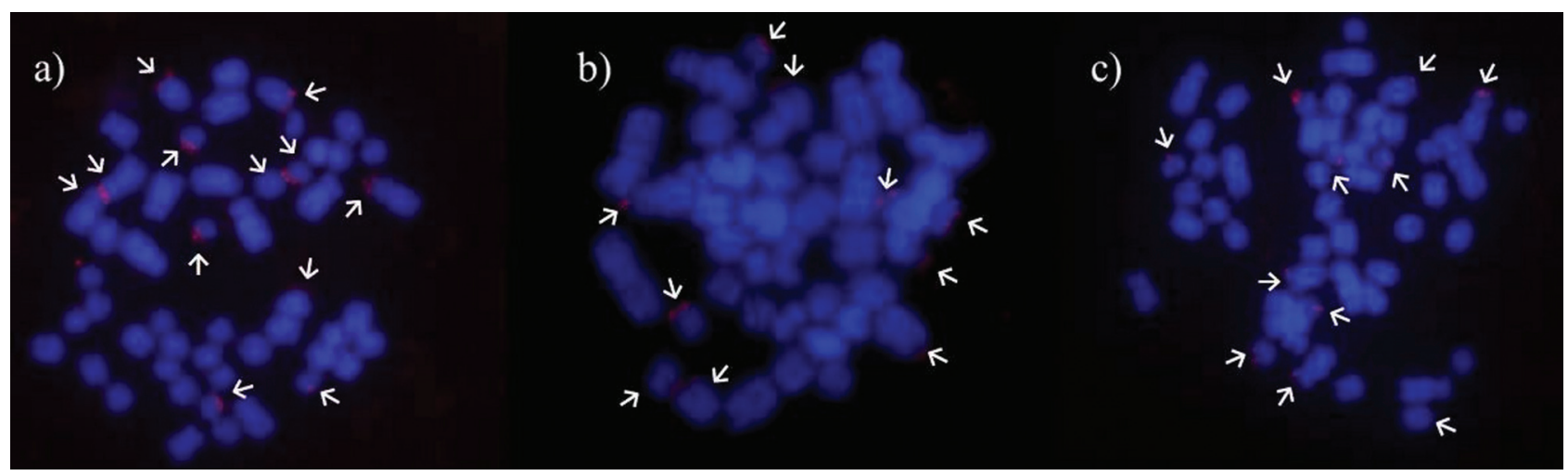

Figure 2 - FISH with the $18 \mathrm{~S}$ rDNA probe. The probe is observed as red signals and the chromosomes were counterstained with DAPI (blue). (A) Baio type $(2 n=50)$ with 12 signals; (B) Carabao breed $(2 n=48)$ with ten signals and $(C)$ crossbreed $(2 n=49)$ with 11 signals. The arrows point to the hybridization signals. 


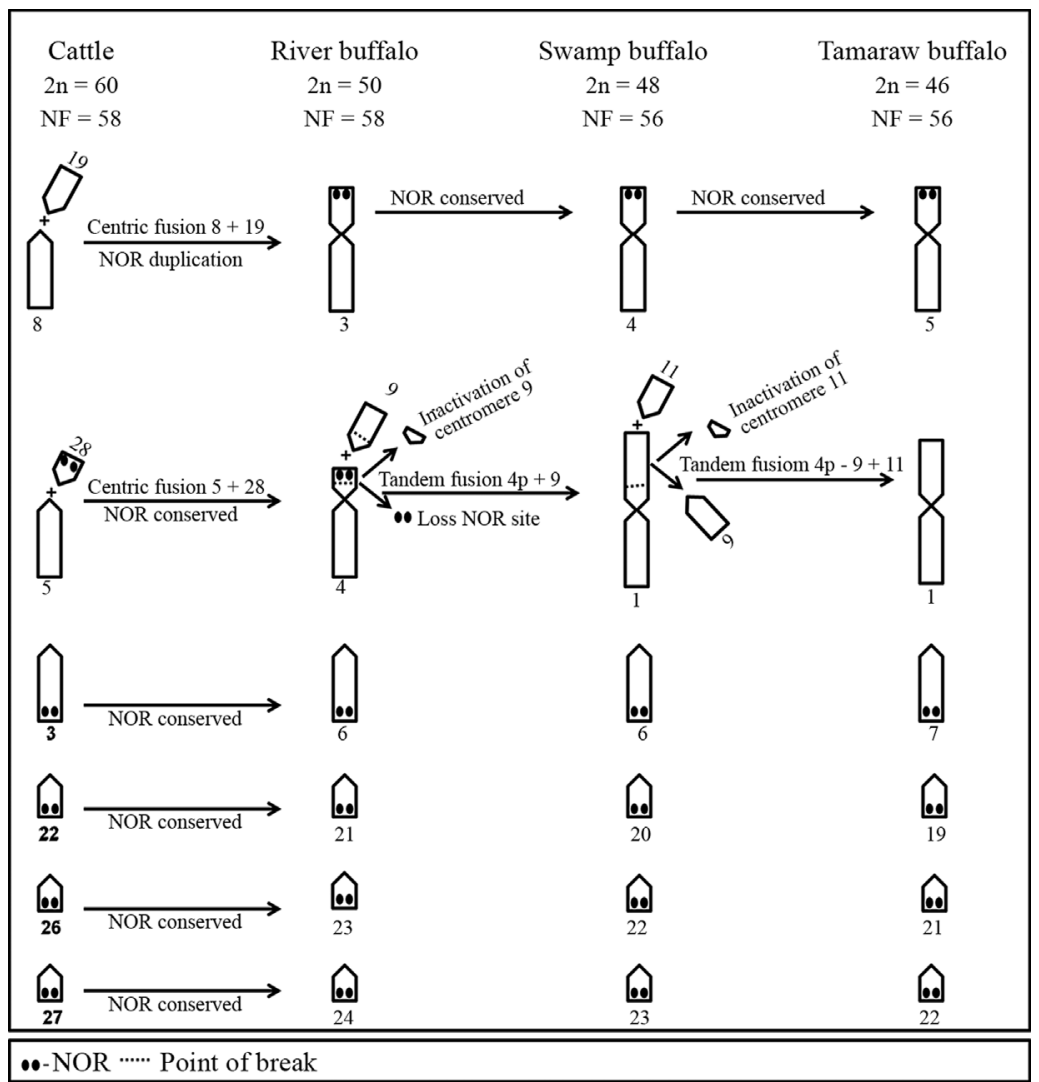

Figure 3 - Schematic representation of the evolution of the NOR-bearing chromosomes in four species of the Bovini tribe. This figure was constructed using the standard GTG-banding ideogram according to Bongso and Hilmi (1982), Iannuzzi et al. (1990) and Tanaka et al. (2000).

served, corresponding to a member of each chromosome pair from their parents: (3p, 6, 20, 22 and 23) in addition to the NOR on the river buffalo $4 \mathrm{p}$. This result was confirmed with the $18 \mathrm{~S}$ ribosomal probe (Figure 2C).

The correct identification of the NOR-bearing chromosomes is critical for karyotypic comparisons among the species. Based on banding homologies, the NOR on chromosome 6 of the domestic buffaloes (Iannuzzi et al., 1996) is the only one shared between species of Bovidae, and is homologous to NORs on chromosome 3 of cattle (Di Berardino et al., 1985), 1p of sheep (Di Meo et al., 1991), 3 of goat (Di Meo et al., 1993) and 7 of the Tamaraw buffalo (Tanaka et al., 2000).

In short, tandem fusions had several important effects in the karyotypic evolution of buffaloes, such as the reduction of the diploid and fundamental numbers, and the loss of NOR sites and interstitial telomeric sequences.

\section{Acknowledments}

Special thanks to Fabiano Torres and Adriana Sassi for the technical support. This work was supported by a scholarship from Coordenação de Aperfeiçoamento de Pessoal de Nível Superior (CAPES) to TMG; and grants from the Universidade Federal do Pampa (Ed. GP 2011 and 2012, PROPESQ/UNIPAMPA) and the Empresa Brasi- leira de Pesquisa Agropecuária (Code SEG 01.06.01.06.00.04, EMBRAPA).

\section{References}

Bongso TA and Hilmi M (1982) Chromosome banding homologies of a tandem fusion in river, swamp and crossbred buffaloes (Bubalus bubalis). Can J Genet Cytol 24:667-673.

de Oliveira EHC, Habermann F, Lacerda O, Sbalqueiro IJ, Wienberg J and Müller S (2005) Chromosome reshuffling in birds of prey: The karyotypes of the world's largest eagle (Harpy eagle, Harpia harpya) compared to that of the chicken (Gallus gallus). Chromosoma 114:338-343.

Di Berardino D, Iannuzzi L, Bettini TM and Matassino D (1981) Ag-NORs variation and banding homologies in two species of Bovidae: Bubalus bubalis and Bos taurus. Can J Genet Cytol 23:89-99.

Di Berardino D, Lioi MB and Iannuzzi L (1985) Identification of nucleolus organizer chromosomes in cattle (Bos taurus L.) by sequential silver staining + RBA banding. Caryologia 38:95-102.

Di Meo GP, Iannuzzi L, Ferrara L and Rubino R (1991) Identification of nucleolus organizer chromosomes in goat (Capra hircus). Caryologia 44:309-316.

Di Meo GP, Iannuzzi L, Perucati A and Ferrara L (1993) Identification of nucleolus organizer chromosomes in sheep (Ovis aries L.). Cytobios 75:183-190. 
Hilmi M and Bongso TA (1983) Nucleolus organizer regions in the chromosomes of river, swamp and crossbred buffaloes [Malaysia]. Philipp J Vet Anim Sci 9:269-270.

Howell WM and Black DA (1980) Controlled silver-staining of nucleolus organizer regions with a protective colloidal developer: A one step method. Experientia 36:1014-1015.

Iannuzzi L (1994) Standard karyotype of the river buffalo (Bubalus bubalis L., $2 \mathrm{n}=50$ ). Report of the committee for the standardization of banded karyotypes of the river buffalo. Cytogenet Cell Genet 67:102-113.

Iannuzzi L and Di Berardino D (1985) Diagrammatic representation of RBA-banded chromosomes of swamp buffalo (Bubalus bubalis L.) and sex chromosome banding homologies with cattle (Bos taurus L.). Caryologia 38:281-295.

Iannuzzi L, Di Meo GP, Perucatti A and Ferrara L (1990) The high resolution G- and R-banding pattern in chromosomes of river buffalo (Bubalus bubalis L.). Hereditas 112:209-215.

Iannuzzi L, Di Meo GP and Perucatti A (1996) Identification of nucleolus organizer chromosomes and frequency of active NORs in river buffalo (Bubalus bubalis 1). Caryologia 49:27-34.

MacEachern S, McEwan J and Goddard M (2009) Phylogenetic reconstruction and the identification of ancient polymorphism in the Bovini tribe (Bovidae, Bovinae). BMC Genomics 10:177.
Marques JRF, Costa MR, Egito AA, Mariante ADS and Albuquerque MSM (2003) Conservation of genetic resources of the small populations of domestic animal of the Amazon Region in Brazil. Anim Genet Resourc Inform 33:31-40.

Moorhead PS, Nowell PC, Mellman WJ, Battips DM and Hungerford DA (1960) Chromosome preparations of leukocytes cultured from human peripheral blood. Exp Cell Res 20:613-616.

Pang CY, Deng TX, Tang DS, Yang CY, Jiang H, Yang BZ and Liang XW (2012) Cloning and sequencing of the rDNA gene family of the water buffalo (Bubalus bubalis). Genet Mol Res 11:2878-2883.

Tanaka K, Matsuda Y, Masangkay JS, Solis CD, Anunciado RVP, Kuro-O M and Namikawa T (2000) Cytogenetic analysis of the Tamaraw (Bubalus mindorensis): A comparison of R-banded karyotype and chromosomal distribution of centromeric satellite DNAs, telomeric sequence, and 18s28 s rRNA genes with domestic water buffaloes. J Hered 9:117-121.

Wurster DH and Benirschke K (1968) Chromosome studies in the superfamily Bovidea. Chromosoma 25:152-171.

Associate Editor: Yatiyo Yonenaga-Yassuda

License information: This is an open-access article distributed under the terms of the Creative Commons Attribution License, which permits unrestricted use, distribution, and reproduction in any medium, provided the original work is properly cited. 